\title{
Improvements in Mapping and Microanalysis by FEG-EPMA
}

\author{
C. M. MacRae ${ }^{1}$, N.C. Wilson ${ }^{1}$ and S. Notoya ${ }^{2}$ \\ ${ }^{1}$ Microbeam Laboratory, CSIRO Minerals, Clayton, Australia, 3168 \\ 2 JEOL Ltd., 1-2 Musashino 3-chome, Akishima, Tokyo 196, Japan
}

The sustained development of electron optics and energy dispersive spectrometry (EDS), together with improvements in beam stability and beam power density have combined to make it easier for the analyst to map a wide variety of materials under a range of beam conditions. We are presently installing a JEOL 8500F field emission microprobe (FEG-EPMA), equipped with two silicon drift EDS spectrometers, an optical spectrometer for cathodoluminescence analysis, and five wavelength dispersive spectrometers (WDS). Both the EDS and optical spectrometers are capable of collecting hyperspectral data sets and here we present some preliminary results from the instrument that includes the ability to combine both spectral EDS and cathodoluminescence with WDS.

The FEG-EPMA is a new generation microanalysis tool, which allows mapping at resolutions of $200 \mathrm{~nm}$ or better over hundreds of microns. To achieve sub micron spatial resolutions stage stepping is generally not employed as mechanical repositioning at these step distances is difficult. A more accurate approach is to step the beam in $\mathrm{X}$ and $\mathrm{Y}$ while collecting $\mathrm{X}$-ray and optical spectra and hence produce a map. However, beam maps cannot be collected over large distances since WDS spectrometers typically only remain in focus over 40 microns. Thus optimum mapping of areas several hundred microns requires a mosaic of beam maps with stage stepping between each. The stage step resolution or reproducibility ultimately determines the uniformity of the pixels at beam map boundaries.

New generation EDS detectors are also making improvements in the collection of mapping data. Ideally, for x-ray microanalysis one wants both ultimate x-ray resolution and x-ray throughput. With the advent of the latest generation of silicon drift detectors (SDD) capable of $140 \mathrm{eV}$ or better on $\mathrm{Mn} ; \mathrm{K \alpha}$ at $100 \mathrm{Kcps}$ it is possible to perform fast mapping and microanalysis [1]. By combining the spectra from two SDD spectrometers count rates in excess of $200 \mathrm{~K}$ cps are expected. By itself this is not enough to solve many mineral processing problems where investigation of characteristics with micron to submicron resolution is required. This type of investigation has traditionally been performed with a high resolution scanning electron microscope equipped with ED spectrometry. The FEG-EPMA should perform this operation faster and, due to the combined EDS and WDS, potential overlapped lines can be negated by utilising the higher resolution of the wavelength dispersive spectrometer.

The determination of valence and structural characteristics can be aided by the inclusion of an optical spectrometer to collect the cathodoluminescence. Commonly employed light optics in electron microprobes are constructed from glass. Glass does not efficiency transmit wavelengths lower than $350 \mathrm{~nm}(3.5 \mathrm{eV})$. To enhance the spectral range the glass optics in this instrument have been replaced with fused quartz, making it possible to extend the transmission of light to $200 \mathrm{~nm}$ $(6.2 \mathrm{eV})$. This opens up the ability to measure transitions in the 3.5-6.2 eV range.

At present it is routine to collect either standalone EDS or cathodoluminescence hyperspectral data sets [2]. In this instrument we are simultaneously collecting hyperspectral datasets from two ED 
spectrometers, a cathodoluminescence system as well as collecting data from the WDS and backscatter detectors. As a result we can collect large volumes of data, however tools for interpreting the data have not kept up with the size of the data collected. Common approaches to data reduction are region of interest selection, principal component analysis [3] and cluster recognition can be employed to reduce the dataset [4,5]. Our implementation of autoclustering creates a cluster tree which can be used to produce a phase map.

The autoclustering partitions the map into distinct phases, and interrogation of the x-ray spectra can reveal the chemical composition of phases but no equivalent procedure exists for cathodoluminescence. We propose that, in some cases, by using a luminescence database one can start to routinely identify the elements, lines and valences giving rise to lines or bands in the optical spectra observed. At present the luminescence database comprises over 900 entries with, 61 minerals and a number of semiconductors. Each entry contains the mineral name, associated formula, charge on the ion, classification as intrinsic or extrinsic, and wavelength of energy of line observed. Where a band has been observed, the peak of the band is given and a full width at half maximum is used to describe the band. Wherever possible the temperature the sample was held at during collection is recorded as well as the type of technique used to excite the luminescence. In addition, the reference for all entries is also recorded so that other characteristics of the lines can be readily checked such as occurrence of mineral, beam conditions or laser conditions, temperature effects, and decay time.

With improvements in both instrumentation and software processing solutions the future of chemical and structural imaging in microanalysis looks bright.

References

[1] T. Schulein and R. Terborg, Microsc. Microanaly. (2004) 11, 460CD

[2] MacRae et al. Microscopy Research and Technique, (2005), vol. 67, number 5, pp 271-277

[3] P. Kotula et al., Microsc. Microanaly. (2003) 9, 1

[4] N. C. Wilson, et al., The16th Australian conference on Electron Microscopy, Canberra, (2000), 46

[5] N.C. Wilson and C.M. MacRae, Microsc. Microanaly. (2005) 11, 434CD 